CDC01-REG1379

\title{
A Coupled Vonlinear Spacecraft Attitude Controller/Observer With an Lnknown Constant Gyro Bias
}

\author{
J. Deutschmann and R.M. Sanner
}

Abstract-A nonlinear control scheme for attitude control of a spacecraft is combined with a nonlinear gyro bias observer for the case of constant gyro bias. The closed loop system is proven to be globally stable, with zero tracking error, thus proving a separation principle for the given system. The nonlinear observer incorporates persistency of excitation, resulting in exponential convergence of the gyro bias error.

\section{Introduction}

Combined observer-controller designs for nonlinear systems are a subject of active research $[1,2,3]$. Successful design of such architectures is complicated by the fact that there is, in general, no separation principle for nonlinear systems. In contrast to linear systems, "certainty equivalence" substitution of the states from even an exponentially converging observer into a nominally stabilizing state feedback control law does not necessarily guarantee stable closed-loop operation for the coupled systems $[2,4,5]$.

In this paper we consider a restricted version of this problem, in particular the task of forcing the attitude of a rigid vehicle to asymptotically track a (time-varying) reference attitude using feedback from sensors with persistent nonzero bias errors. Specifically, we propose utilizing an angular velocity observer from $[2,3]$ in a certainty equivalence fashion with the nonlinear control law proposed in [6], and demonstrate in this case that the resulting system results in stable closed-loop operation, with asymptotically perfect tracking.

The proof proceeds in two steps. First, we extend the analysis of the observer in $[2,3]$ to the case of constant gyro bias, and use a persistency of excitation argument to demonstrate that the bias estimates provided by this observer are in fact exponentially convergent to the true bias values. The proof in [2] uses a Lyapunov argument to obtain a similar result, but under the assumption that the biases themselves are exponentially decaying; this restriction is removed here. Second, we consider the certainty equivalence use of these observer estimates in the nonlinear feedback control algorithm proposed in [6] and show that the perturbation introduced by this strategy into the closed-loop dynamics can be represented as a bounded function of the vehicle states multiplying the observer transients. Quantifying the impact these perturbations have on the Lyapunov

\footnotetext{
Julie Deutschmann is with the Flight Dynamics. Analysis Branch at the NASA Goddard Space Flight Center, Greenbelt. MD 207\%1

Robert M. Sanner is with the Department of Acrospace Engineering at the Lniversity of Maryland, College Park, Maryland 20742
} 
analysis given in [6], we demonstrate that the stability and convergence properties of the controller are, in fact, maintained in the face of the perturbations, completing our analysis of the proposed methodology.

The paper is organized as follows. Section II contains definitions of the terms used in the controller and observer. In Section III the nonlinear observer for the constant gyro bias is developed and the exponential convergence is proven. Section IV presents the nonlinear controller design and the proof of stability of the closed loop system and the convergence of the tracking errors. Section $V$ presents simulation results, followed by conclusions in Section VI.

\section{Definitions}

The attitude of a spacecraft can be represented by a four component quaternion, consisting of a rotation angle and unit rotation vector, known as the Euler axis

$$
\mathbf{q}=\left[\begin{array}{l}
\mathbf{e} \sin \frac{\phi}{2} \\
\cos \frac{\phi}{2}
\end{array}\right]=\left[\begin{array}{l}
\varepsilon \\
\eta
\end{array}\right]
$$

where $\phi$ is the rotation angle, $\mathrm{e}$ is the Euler axis, $\varepsilon$ and $\eta$ are the vector and scalar portions of the quaternion, respectively. Note that $\|q\|=1$ by definition. The quaternion represents the rotation from an inertial coordinate system to the spacecraft body coordinate system. A rotation matrix can be computed from the quatemion as [6]

$$
R(q)=\left(\eta^{2}-\varepsilon^{T} \varepsilon\right) I+2 \varepsilon \varepsilon^{\top}-2 \eta S(\varepsilon)
$$

where $S(\varepsilon)$ is a cross product matrix formed from the vector $\varepsilon$.

$$
S(\varepsilon)=\left[\begin{array}{ccc}
0 & -\varepsilon_{z} & \varepsilon_{y} \\
\varepsilon_{z} & 0 & -\varepsilon_{x} \\
-\varepsilon_{y} & \varepsilon_{x} & 0
\end{array}\right]
$$

A desired target attitude is represented by the quaternion, $\mathbf{q}_{s}^{\top}=\left[\varepsilon_{\mathrm{j}}^{\top}, \eta_{d}\right]$. The attitude error used in the controller is defined as a rotation from the desired body frame to the actual body frame and is computed according to [7]

$$
\widetilde{\mathbf{q}}_{\mathrm{c}}=\left[\begin{array}{c}
\widetilde{\varepsilon}_{\mathrm{c}} \\
\tilde{\eta}_{\mathrm{c}}
\end{array}\right]=\mathbf{q} \otimes \mathrm{q}_{\mathrm{d}}{ }^{-1}=\left[\begin{array}{cc}
\eta_{\mathrm{d}} I-S\left(\varepsilon_{\mathrm{s}}\right) & -\varepsilon_{\mathrm{d}} \\
\varepsilon_{\mathrm{d}}{ }^{\mathrm{s}} & \eta_{\mathrm{d}}
\end{array}\right]\left[\begin{array}{l}
\varepsilon \\
\eta
\end{array}\right]
$$

Similarly, in the observer, the attitude error is defined as the rotation from the estimated body frame to the actual body frame as 


$$
\widetilde{\mathbf{q}}_{o}=\left[\begin{array}{l}
\widetilde{\varepsilon}_{o} \\
\tilde{\eta}_{o}
\end{array}\right]=\mathbf{q} \otimes \hat{\mathbf{q}}^{-1}=\left[\begin{array}{cc}
\hat{\eta}[-S(\hat{\varepsilon}) & -\hat{\varepsilon} \\
\hat{\varepsilon}^{\top} & \hat{\eta}
\end{array}\right]\left[\begin{array}{l}
\varepsilon \\
\eta
\end{array}\right]
$$

where $\hat{\mathbf{q}}$ represents the attitude state of the observer. Note that $\widetilde{\varepsilon}_{\mathrm{c}}=0, \widetilde{\eta}_{\mathrm{c}}= \pm 1$ indicates that the spacecraft is aligned with the desired attitude and similarly, $\widetilde{\varepsilon}_{o}=0, \widetilde{\eta}_{o}= \pm 1$ indicates that the attitude estimate is aligned with the actual attitude.

The kinematics equation for the quaternion is given as

$$
\dot{\mathbf{q}}=\left[\begin{array}{c}
\dot{\varepsilon} \\
\dot{\eta}
\end{array}\right]=\frac{1}{2}\left[\begin{array}{c}
\eta I+S(\varepsilon) \\
-\varepsilon^{\top}
\end{array}\right] \omega=\frac{1}{2} Q \omega
$$

where $\omega$ is the spacecraft angular velocity. The angular velocity is typically measured by a gyro, which can be corrupted with both systematic and random errors. In this work we consider only the case of systematic errors. In the case of a gyro bias, the angular velocity can be written as

$$
\omega=\omega_{g}+\mathbf{b}
$$

where $\omega_{\mathrm{g}}$ is the angular velocity from the gyro and $\mathbf{b}$ is the gyro bias. An estimate of the angular velocity is given as $\hat{\omega}=\omega_{g}+\hat{\mathbf{b}}$. The bias error is defined as the difference between the true and estimated bias

$$
\widetilde{b}=\mathbf{b}-\hat{\mathbf{b}}
$$

Finally, a measure of the discrepancy between the actual and desired angular velocity in the controller is computed as [8]

$$
\widetilde{\omega}_{\mathrm{c}}=\omega-\mathrm{R}\left(\widetilde{\mathbf{q}}_{\mathrm{c}}\right) \omega_{\mathrm{d}}
$$

which is defined such that $\dot{\tilde{\mathbf{q}}}_{\mathrm{c}}=\frac{1}{2} \mathrm{Q}\left(\widetilde{\mathbf{q}}_{\mathrm{c}}\right) \widetilde{\boldsymbol{\omega}}_{\mathrm{c}}$.

\section{Nonlinear Observer for Constant Gyro Bias}

Following the development of [2] a state observer for the bias can be defined as

$$
\begin{gathered}
\dot{\hat{\mathbf{q}}}=\frac{1}{2} \hat{\mathrm{Q}} \mathrm{R}^{\mathrm{T}}\left(\widetilde{\mathbf{q}}_{\mathrm{o}}\right)\left(\omega_{\mathrm{g}}+\hat{\mathbf{b}}+k \widetilde{\varepsilon}_{\mathrm{o}} \operatorname{sgn}\left(\widetilde{\eta}_{\mathrm{o}}\right)\right) \\
\dot{\hat{\mathbf{b}}}=\frac{1}{2} \widetilde{\varepsilon}_{\mathrm{o}} \operatorname{sgn}\left(\widetilde{\eta}_{\mathrm{o}}\right)
\end{gathered}
$$


The gain, $k$, is chosen as a positive constant. The $R^{\top}\left(\widetilde{\mathbf{q}}_{v}\right)$ resolves the angular velocity terms in the observer frame.

Computing the derivatives of $\widetilde{q}_{0}$ in (1) and $\widetilde{\mathbf{b}}$ in (2) results in the following differential error equations

$$
\begin{gathered}
\dot{\tilde{\mathbf{q}}}_{0}=\left[\begin{array}{c}
\dot{\varepsilon}_{0} \\
\dot{\tilde{\eta}}_{0}
\end{array}\right]=\frac{1}{2}\left[\begin{array}{c}
\widetilde{\eta}_{0} I+S\left(\widetilde{\varepsilon}_{0}\right) \\
-\widetilde{\varepsilon}_{0}{ }^{T}
\end{array}\right]\left[\widetilde{\mathbf{b}}-k \widetilde{\varepsilon}_{0} \operatorname{sgn}\left(\widetilde{\eta}_{0}\right)\right] \\
\dot{\widetilde{\mathbf{b}}}=-\frac{1}{2} \widetilde{\varepsilon}_{0} \operatorname{sgn}\left(\widetilde{\eta}_{0}\right)
\end{gathered}
$$

The equilibrium states are $(0,0,0, \pm 1,0,0,0)$. Again, following the development of [2], a Lyapunov function is chosen as

$$
V_{o}=\frac{1}{2} \widetilde{\mathbf{b}}^{\mathrm{T}} \widetilde{\mathbf{b}}+\frac{1}{2} \begin{cases}\left(\widetilde{\eta}_{0}-1\right)^{2}+\widetilde{\boldsymbol{\varepsilon}}_{0}^{\mathrm{T}} \widetilde{\boldsymbol{\varepsilon}}_{0} & \widetilde{\eta} \geq 0 \\ \left(\widetilde{\eta}_{0}+1\right)^{2}+\widetilde{\boldsymbol{\varepsilon}}_{0}^{\top} \widetilde{\boldsymbol{\varepsilon}}_{0} & \tilde{\eta}_{0}<0\end{cases}
$$

Taking the derivative of $V_{0}$, and noting that $\widetilde{\varepsilon}_{0} \dot{\widetilde{\varepsilon}}_{0}+\widetilde{\eta}_{0} \dot{\tilde{\eta}}_{0}=0$, yields

$$
\dot{V}_{0}=\widetilde{\mathbf{b}}^{\top} \dot{\overrightarrow{\mathbf{b}}}+\left\{\begin{array}{cc}
-\dot{\tilde{\eta}}_{0} & \tilde{\eta}_{0} \geq 0 \\
\dot{\vec{\eta}}_{0} & \tilde{\eta}_{0}<0
\end{array}\right.
$$

Substituting $\dot{\tilde{\eta}}_{\mathrm{o}}$ and $\dot{\overrightarrow{\mathrm{b}}}$ from above results in

$$
\dot{V}_{0}=-\frac{k_{1}}{2} \widetilde{\varepsilon}_{0}^{\top} \widetilde{\varepsilon}_{0} \leq 0
$$

This establishes that $\widetilde{b}, \widetilde{\varepsilon}_{0}$, and $\widetilde{\eta}_{0}$ are globally uniformly bounded; a further application of Barbalat's lemma [4] shows that, in fact, $\widetilde{\varepsilon}_{0} \rightarrow 0$.

Since all the signals in the observer have been demonstrated to be bounded, $\dot{\widetilde{\varepsilon}}_{\mathrm{o}}$ and $\dot{\widetilde{\mathbf{b}}}$ can be analyzed as

$$
\dot{\mathbf{x}}(\mathrm{t})=\mathrm{A}(\mathrm{t}) \mathbf{x}(\mathrm{t})
$$

and the derivative of the Lyapunov function written as

$$
\dot{V}_{o}=-x^{\top} C^{\top} C x \leq 0
$$

where 


$$
\begin{gathered}
\mathbf{x}(\mathrm{t})=\left[\begin{array}{c}
\widetilde{\varepsilon}_{0}(t) \\
\widetilde{\mathbf{b}}(\mathrm{t})
\end{array}\right] ; A(t)=\left[\begin{array}{cc}
-\frac{1}{2} k_{1} \operatorname{sgn}\left(\tilde{\eta}_{0}(t)\right)\left(\widetilde{\eta}_{0}(t) I+S\left(\widetilde{\varepsilon}_{0}(t)\right)\right) & \left(\widetilde{\eta}_{0}(t) I+S\left(\widetilde{\varepsilon}_{0}(t)\right)\right) \\
\frac{1}{2} \operatorname{sgn}\left(\tilde{\eta}_{0}(t)\right) I & 0
\end{array}\right] ; \\
C=\left[\sqrt{\frac{k}{2}} I \quad 0\right]
\end{gathered}
$$

By a standard argument (e.g. Theorem 4.5 of [4]), the equilibrium point $\mathbf{x}=0$ of this system is exponentially stable if the pair $(A(t), C)$ is uniformly completely observable (UCO). Since observability properties are unchanged under output feedback, this will be true if and only if the pair $(A(t)-K(t) C, C)$ is uniformly observable, with $K(t)$ piecewise continuous and bounded. With $\mathrm{K}(\mathrm{t})$ chosen as

$$
K(t)=\left[\begin{array}{c}
-\sqrt{\frac{k}{2}} \operatorname{sgn}\left(\widetilde{\eta}_{0}\right)\left(\tilde{\eta}^{\prime} I+S\left(\widetilde{\varepsilon}_{0}\right)\right) \\
-\sqrt{\frac{1}{2 k}} \operatorname{sgn}\left(\widetilde{\eta}_{0}\right) I
\end{array}\right]
$$

The matrix $(A(t)-K(t) C)$ becomes

$$
A(t)-K(t) C=\left[\begin{array}{cc}
0 & \frac{1}{2}\left(\tilde{\eta}_{0} I+S\left(\widetilde{\varepsilon}_{0}\right)\right) \\
0 & 0
\end{array}\right]
$$

The state transition matrix for $(6)$ is

$$
\Phi(\tau, t)=\left[\begin{array}{cc}
I & \frac{1}{2} \Sigma(\tau, t) \\
0 & I
\end{array}\right]
$$

with $\frac{1}{2} \Sigma(\tau, \mathrm{t})=\frac{1}{2} \int_{t}^{\tau} \mathrm{Q}_{1}\left(\widetilde{\mathrm{q}}_{\mathrm{o}}(\sigma)\right) \mathrm{d} \sigma=\frac{1}{2} \int_{\mathrm{t}}^{\tau}\left(\widetilde{\eta}_{\mathrm{o}}(\sigma) I+\mathrm{S}\left(\widetilde{\boldsymbol{\varepsilon}}_{\mathrm{o}}(\sigma)\right)\right) \mathrm{d} \sigma$. The observability Gramian for the pair $(A(t)-K(t) C, C)$ is given by

$$
W(t+T)=\int_{t}^{t+T} \Phi^{T}(\tau, t) C^{T} C \Phi(\tau, t) d \tau=\int_{t}^{t+T}\left[\begin{array}{cc}
\frac{k}{2} I & \frac{k}{t} \Sigma(\tau, t) \\
\frac{k}{t} \Sigma^{T}(\tau, t) & \frac{k}{+8} \Sigma^{T}(\tau, t) \Sigma(\tau, t)
\end{array}\right] d \tau
$$

and the system is UCO if there exists a T $>0$ and positive constants $k_{l}>0, k_{2}>0$ such that, for all $\mathrm{t} \geq \mathrm{t}_{0}$

$$
k_{1} I \geq W(t+T) \geq k_{2} I
$$

Úsing Lemma $13 .+$ of $[j]$, this property is assured for $(7)$ if $Q_{1}(t)$ and $\dot{Q}_{1}(t)$ are bounded, and there are positive constants $T_{2}, \alpha_{1}$ and $\alpha_{2}$ such that, for all $t \geq t_{1}$ ) 


$$
\alpha_{2} I \geq \int_{1}^{t+T_{2}} Q_{1}(\tau) Q_{1}^{\top}(\tau) d \tau \geq \alpha_{1} I
$$

The upper bound is satisfied since $Q_{1}(t)$ is bounded. To determine if the lower bound is satisfied, rewrite the matrix $Q_{1}(t) Q_{1}{ }^{\top}(t)$ as

$$
Q_{1}(t) Q_{1}{ }^{T}(t)=\left(\widetilde{\eta}_{0}(t) I+S\left(\widetilde{\varepsilon}_{0}(t)\right)\left(\widetilde{\eta}_{0}(t) I-S\left(\widetilde{\varepsilon}_{0}(t)\right)=I-\varepsilon_{0}(t) \varepsilon_{0}(t)^{T}\right.\right.
$$

Since $\widetilde{\varepsilon}_{0} \rightarrow 0$ asymptotically, for any $\delta>0$, there exists a $T_{1}(\delta)>0$ such that $\left\|\widetilde{\varepsilon}_{0}\right\|<\delta$ for all $t \geq t_{0}+T_{1}$. Taking any $\delta<1$ and $T_{2}>T_{1},(9)$ implies that

$$
\mathbf{z}^{\mathrm{T}}\left[\int_{t}^{t+T_{2}} Q_{1}(\tau) Q_{1}{ }^{T}(\tau) d \tau\right] \mathbf{z}=(1-\delta)\left(T_{2}-T_{1}\right)\|z\|^{2}
$$

for any $z$ in $R^{6}$ and thus (8) is satisfied. This demonstrates the required UCO property for the observer, and hence establishes that $\widetilde{\boldsymbol{\varepsilon}}_{\mathrm{o}}$ and $\widetilde{\mathbf{b}}$ approach zero exponentially fast.

\section{Nonlinear Controller Design}

The complete attitude dynamics for a rigid spacecraft are given as

$$
\begin{gathered}
\mathrm{H} \dot{\boldsymbol{\omega}}-\mathrm{S}(\mathrm{H} \omega) \boldsymbol{\omega}=\mathbf{u} \\
\dot{\mathbf{q}}=\frac{1}{2} \mathrm{Q}(\mathbf{q}) \boldsymbol{\omega}
\end{gathered}
$$

where $\mathrm{H}$ is a constant, symmetric inertia matrix and $\mathbf{u}$ is the applied external torque, for example, from attached rocket thrusters. The goal of the controller is for the actual attitude $q(t)$ to asymptotically track a (generally) time-varying desired attitude $q_{d}(t)$ and angular velocity $w_{\mathrm{J}}(t)$, related for consistency by $\dot{\mathbf{q}}_{d}=\frac{1}{2} \mathrm{Q}\left(\mathbf{q}_{\mathrm{d}}\right) \omega_{\mathrm{d}}$. It is assumed that $w_{d}(t)$ is bounded and differentiable with $\dot{\omega}_{d}(t)$ also bounded.

The passivity based controller of [6] utilizes the composite error metric

$$
s=\widetilde{\omega}_{c}+\lambda \widetilde{\varepsilon}_{c}=\omega-\omega_{r}
$$

Where from (3), $\omega_{\mathrm{r}}=\mathrm{R}\left(\widetilde{\mathbf{q}}_{\mathrm{c}}\right) \omega_{\mathrm{d}}-\lambda \widetilde{\varepsilon}_{\mathrm{c}}$. Taking the derivative of (10) and multiplying by $\mathrm{H}$ results in

$$
H \dot{s}=H \dot{\omega}-H \dot{\omega}_{r}=\mathbf{u}+S(H \omega) \omega-H \alpha_{r}
$$

where

$$
\alpha_{r}=\dot{\omega}_{r}=R\left(\widetilde{\mathbf{q}}_{i}\right) \dot{\omega}_{j}-S\left(\widetilde{\omega}_{i}\right) R\left(\widetilde{q}_{i}\right) \omega_{j}-\dot{\lambda}_{i} Q_{i}\left(\widetilde{q}_{i}\right) \widetilde{\omega}_{i}
$$


and $Q_{1}\left(\widetilde{q}_{c}\right)=\widetilde{\eta}_{c} I+S\left(\widetilde{\varepsilon}_{c}\right)$ as defined above. With these definitions, the control law

$$
\mathbf{u}=-\mathrm{K}_{\mathrm{D}} \mathrm{s}+\mathrm{H} \alpha_{\mathrm{r}}-\mathrm{S}(\mathrm{H} \omega) \omega_{\mathrm{r}}
$$

for any symmetric, positive definite matrix $K_{D}$ as shown in [6] produces the desired stability and asymptotic tracking properties.

In the current application, the control law (12) cannot be implemented because exact measurements of the angular velocity $\omega$ are not available. Instead a certainty equivalence approach is employed using the estimates $\hat{\omega}$ from above, resulting in

$$
\mathbf{u}=-K_{D} \hat{s}+H \hat{\alpha}_{r}-S(H \hat{\omega}) \omega_{r}
$$

where $\hat{\mathbf{s}}=\hat{\boldsymbol{\omega}}-\omega_{\mathrm{r}}, \hat{\widetilde{\omega}}_{\mathrm{c}}=\hat{\boldsymbol{\omega}}-\mathrm{R}\left(\widetilde{\mathrm{q}}_{\mathrm{c}}\right) \omega_{\mathrm{d}}$, and

$$
\hat{\boldsymbol{\alpha}}_{\mathrm{r}}=\mathrm{R}\left(\widetilde{\mathbf{q}}_{\mathrm{c}}\right) \dot{\boldsymbol{\omega}}_{\mathrm{d}}+\mathrm{S}\left(\mathrm{R}\left(\widetilde{\mathbf{q}}_{\mathrm{c}}\right) \omega_{\mathrm{d}}\right) \dot{\tilde{\omega}}_{\mathrm{c}}-\lambda \mathrm{Q}_{\mathrm{t}}\left(\widetilde{\mathbf{q}}_{\mathrm{c}}\right) \hat{\tilde{\boldsymbol{\omega}}}_{\mathrm{c}} .
$$

Substituting (13) into (11), along with (10), and noting that $\widetilde{\mathbf{s}}=\mathbf{s}-\hat{\mathbf{s}}=\widetilde{\mathbf{b}}$, $\widetilde{\boldsymbol{\alpha}}_{\mathrm{r}}=\left[\mathrm{S}\left(\mathrm{R}\left(\widetilde{\mathbf{q}}_{\mathrm{c}}\right) \omega_{\mathrm{d}}\right)-\lambda \mathrm{Q}_{1}\left(\widetilde{\mathbf{q}}_{\mathrm{c}}\right)\right] \widetilde{\mathbf{b}}$, and $\widetilde{\omega}_{\mathrm{c}}-\tilde{\widetilde{\omega}}_{\mathrm{c}}=\omega-\hat{\boldsymbol{\omega}}=\widetilde{\mathbf{b}}$ produces the closed-loop dynamics

$$
H \dot{\mathbf{s}}-\mathrm{S}(H \omega) \mathbf{s}+\mathrm{K}_{\mathrm{D}} \mathbf{s}=\left[-\mathrm{S}\left(\boldsymbol{\omega}_{\mathrm{r}}\right) \mathrm{H}-\mathrm{HS}\left(\mathrm{R}\left(\widetilde{\mathbf{q}}_{\mathrm{c}}\right) \boldsymbol{\omega}_{\mathrm{d}}\right)+\lambda H \mathrm{Q}_{1}\left(\widetilde{\mathbf{q}}_{\mathrm{c}}\right)+\mathrm{K}_{\mathrm{D}}\right] \widetilde{\mathbf{b}}
$$

The terms on the right hand side of (14) can be rewritten as $\Delta\left(\widetilde{\mathbf{q}}_{\mathrm{c}}, \omega_{\mathrm{d}}\right) \widetilde{\mathbf{b}}$. Since $\left\|\widetilde{\mathbf{q}}_{\mathrm{c}}\right\|=1$ by definition and $\left\|\omega_{\mathrm{d}}\right\|<\infty$ by assumption

$$
\gamma \equiv \sup _{t \geq t_{0}} \sup _{\left.\mid \widetilde{q}_{c}\right\}=1} \| \Delta\left(\widetilde{\mathbf{q}}_{c}, \omega_{d}(t) \|<\infty\right.
$$

Using the Lyapunov function $V_{c}=\frac{1}{2} s^{\top} H s$, the derivative of $V_{c}$ along closed-loop trajectories of (14) satisfies the inequality

$$
\dot{V}_{c}=-s^{\top} K_{D} s+s^{\top} \Delta \widetilde{b} \leq-k_{D}\|\hat{s}\|^{2}+\gamma\|s\|\|\widetilde{b}\|
$$

where $k_{D}$ is the smallest eigenvalue of $K_{D}$. Using Young's inequality [5] on the last term above, $\dot{V}_{c}$ is rewritten as

$$
\dot{V}_{c} \leq-\frac{k_{D}}{2}\|s\|^{2}+\frac{\gamma^{2}}{2 k_{D}}\|\tilde{\mathbf{b}}\|^{2}
$$


Thus, recalling from the observer analysis that $\|\tilde{b}\|$ is bounded, $\mathbf{s}$ is also seen to be uniformly bounded. Similarly $\dot{\boldsymbol{s}}$ is uniformly bounded, since all the terms in (14) have been demonstrated to be bounded. Moreover, $s \in \mathrm{L}_{2}$ since for any $\mathrm{t} \geq \mathrm{t}_{0}$

$$
\int_{t_{0}}^{t}\|s(\tau)\|^{2} d \tau \leq \frac{2}{k_{D}}\left[V_{c}(t)-V_{c}\left(t_{0}\right)\right]+\frac{\delta^{2}}{4 k_{D}^{2}} \int_{t_{0}}^{t}\|\widetilde{b}(\tau)\|^{2} d \tau
$$

The integral on the right hand side of $(15)$ is finite for any $t>t_{0}$ since $\|\tilde{\mathbf{b}}\|$ is converging exponentially to zero. Thus $s \in \mathrm{L}_{\infty} \cap \mathrm{L}_{2}, \dot{s} \in \mathrm{L}_{\infty}$, hence by Barbalat's lemma [5] $\lim _{t \rightarrow \infty} s(t)=0$.

This establishes convergence of the composite metric $s$ to zero; now the convergence of the actual attitude enror $\widetilde{\varepsilon}_{\mathrm{c}}$ is examined. From (10), $\|s\|^{2}=\left\|\widetilde{\omega}_{c}\right\|^{2}+2 \lambda \widetilde{\omega}_{\mathrm{c}}^{\mathrm{T}} \widetilde{\boldsymbol{\varepsilon}}_{\mathrm{c}}+\lambda^{2}\left\|\widetilde{\boldsymbol{\varepsilon}}_{\mathrm{c}}\right\|^{2}$. Since $\|\widetilde{\varepsilon}\|_{\mathbb{C}}$ is bounded by definition, $s \in \mathrm{L}_{\infty}$ implies that $\widetilde{\omega}_{\mathfrak{c}} \in \mathrm{L}_{\infty}$. This in turn demonstrates that $\dot{\widetilde{\varepsilon}}_{\mathrm{c}} \in \mathrm{L}_{\infty}$ since $\dot{\widetilde{\mathrm{q}}}_{\mathrm{c}}=\frac{1}{2} \mathrm{Q}\left(\widetilde{\mathrm{q}}_{\mathrm{c}}\right) \widetilde{\boldsymbol{\omega}}_{\mathrm{c}}$ and both terms on the right are bounded. Finally, $\widetilde{\varepsilon}_{c}$ is also in $L_{2}$ since for any $t \geq t_{0}$

$$
\int_{t_{0}}^{t}\left\|\widetilde{\varepsilon}_{\mathrm{c}}(\tau)\right\|^{2} \mathrm{~d} \tau \leq \frac{1}{\lambda^{2}} \int_{t_{0}}^{t}\|\mathbf{S}(\tau)\|^{2} \mathrm{~d} \tau-\frac{2}{\lambda} \int_{t_{0}}^{t} \widetilde{\omega}_{\mathrm{c}}^{\mathrm{T}} \widetilde{\mathbf{\varepsilon}}_{\mathrm{c}} \mathrm{d} \tau
$$

noting that $\widetilde{\omega}_{\mathrm{c}}^{\mathrm{T}} \widetilde{\varepsilon}_{\mathrm{c}}=2 \dot{\tilde{\eta}}_{\mathrm{c}}$, the terms on the right of (16) are finite. Thus, $\widetilde{\varepsilon}_{c} \in L_{\infty} \cap L_{2}, \dot{\widetilde{\varepsilon}}_{c} \in L_{x}$ and Barbalat again implies that $\lim _{\mathrm{t} \rightarrow \infty} \widetilde{\varepsilon}_{c}=0$. (Note that an analogous argument establishes that $\lim _{t \rightarrow \infty} \widetilde{\omega}_{c}=0$.)

\section{Simulation Results}

The spacecraft attitude controller/observer design is tested with a Matlab simulation. The inertia matrix is a diagonal matrix with principal moments of inertia of $[90,100,70]^{\mathrm{T}}$ $\mathrm{kg}-\mathrm{m}^{2}$. The desired angular velocity is $\omega_{d}{ }^{\top}=[0,0.11,0] \mathrm{rad} / \mathrm{sec}$ and $\mathbf{q}_{\mathrm{d}}{ }^{\top}=[0,0,0,1]^{\mathrm{T}}$. Table I lists the initial conditions for the observer and controller, as well as the true gyro bias and true initial angular velocity. The gains are chosen as $k=1, K_{D}=k_{D} I$ with $k_{D}=6$.

Table I. Simulation Initial values

\begin{tabular}{cc} 
Variable & Initial Value \\
\hline $\mathbf{q}$ & {$[0,1,0,0]^{\mathrm{T}}$} \\
$\mathbf{q}$ & {$[0,0,1,0]^{\mathrm{T}}$} \\
$\omega-$ true & {$[-.1,2,-.4]^{\mathrm{T}} \mathrm{rad} / \mathrm{sec}$} \\
$\mathbf{b}-$ true & {$[.05,-0.05,0.033]^{\mathrm{T}}$ rad sec }
\end{tabular}




$$
\hat{\mathbf{b}} \quad[0,0,0]^{\mathrm{T}} \mathrm{rad} / \mathrm{sec}
$$

Figures 1 and 2 show that $\widetilde{\varepsilon}_{0}$ and $\widetilde{\mathbf{b}}$, respectively, converge to zero. Figures 3 and 4 show that $\widetilde{\varepsilon}_{\mathrm{c}}$ and $\widetilde{\omega}$, respectively, also converge to zero.

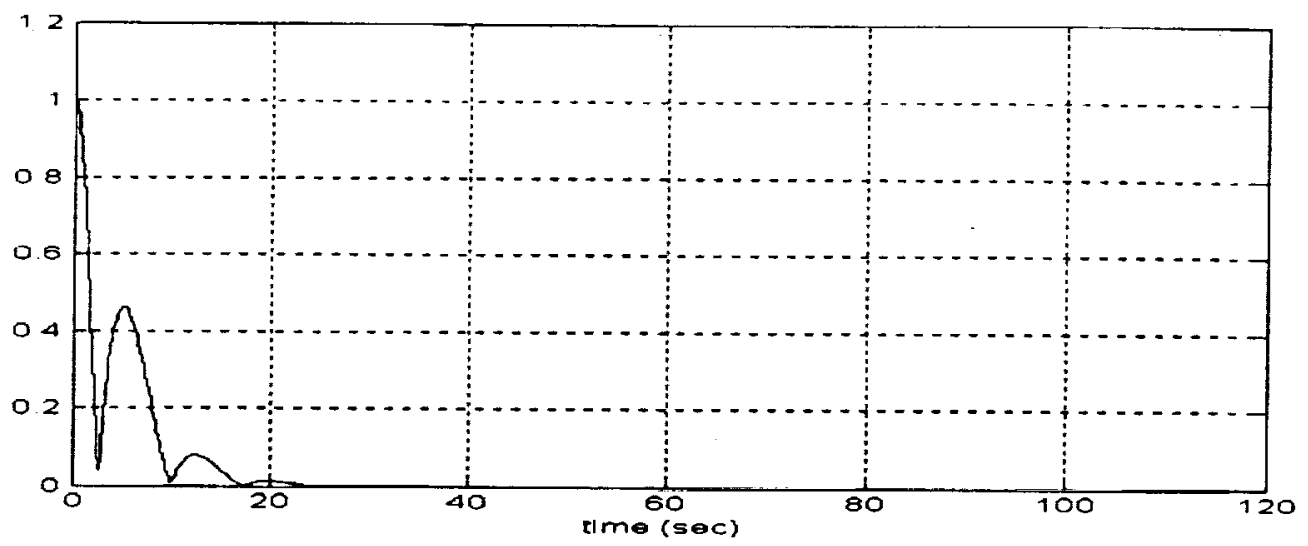

Figure 1. Observer Error - $\left\|\widetilde{\varepsilon}_{0}\right\|_{2}$

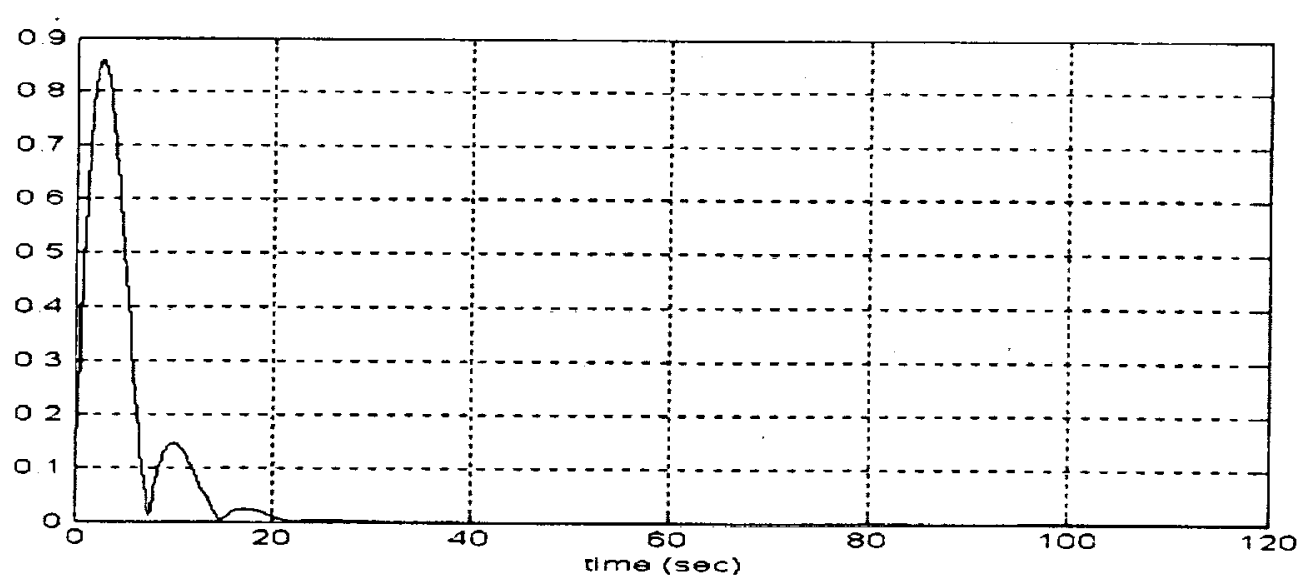

Figure 2. Observer Error - $\|\widetilde{\mathrm{b}}\|_{2}$

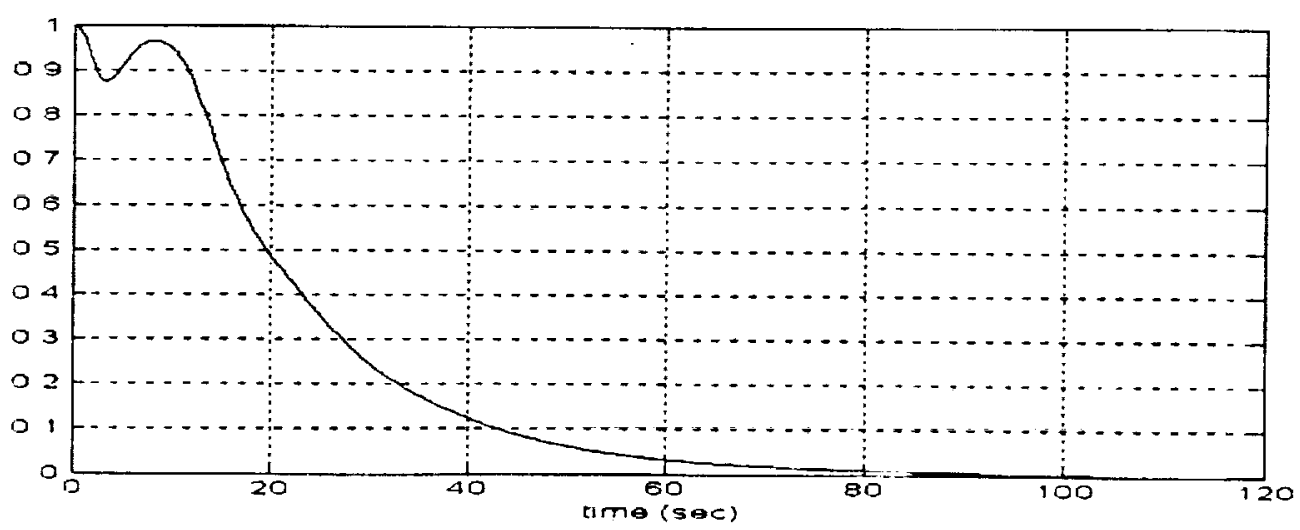

Figure 3. Controller Error $\cdot\left\|\widetilde{\varepsilon}_{\mathrm{c}}\right\|_{2}$ 


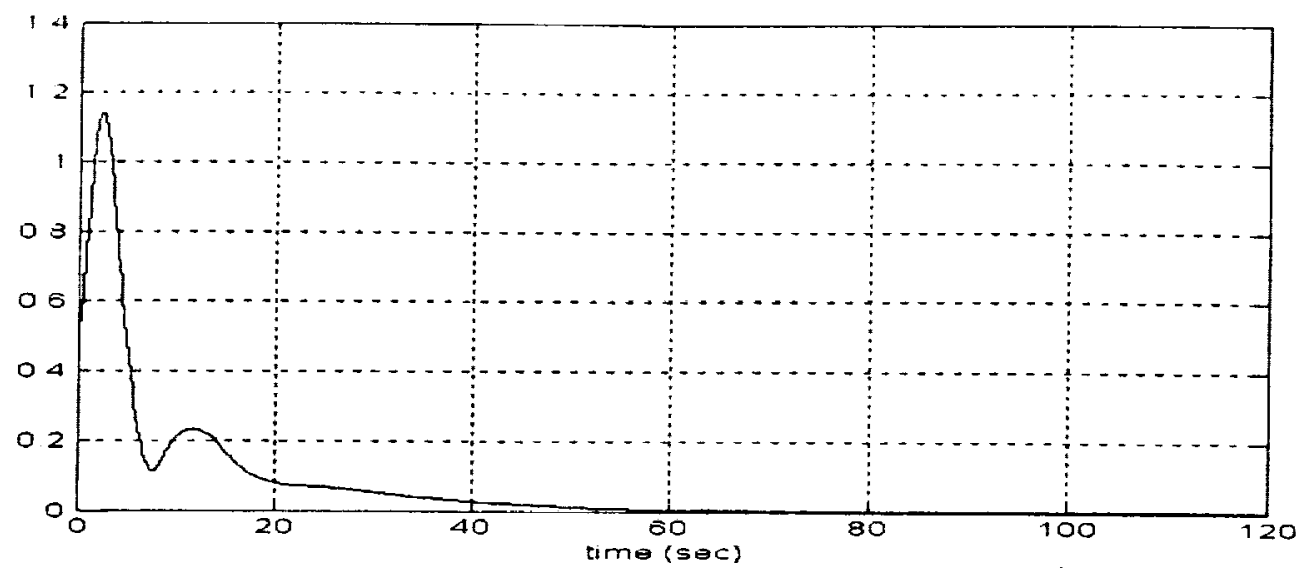

Figure 4. Controller Error - $\widetilde{\omega}$

\section{Conclusions}

A nonlinear controller/observer is presented for spacecraft attitude applications, given a constant gyro bias. The nonlinear observer is globally convergent, with identification of the gyro bias proven through a persistency of excitation argument. The nonlinear controller is a passivity based controller. The control input requires the use of the gyro bias estimate from the nonlinear observer. The closed loop stability properties of this nonlinear controller coupled with the nonlinear observer are analyzed and the system is found to be globally stable, leading to a separation principle for the nonlinear system. Given a desired target attitude and target angular velocity, this system guarantees zero tracking error.

\section{References}

[1] S. Salcudean, "A Globally Convergent Angular Velocity Observer for Rigid Body Motion", IEEE Transactions on Automatic Control, Vol. 36, No. 12, December, 1991.

[2] H. Nijmeijer and T.I. Fossen, Lecture Notes in Control and Information Sciences, New Directions in Nonlinear Observer Design, Springer-Verlag, 1999, pp. 135159.

[3] R. Alonso, J.L. Crassidis, and J.L. Junkins, "Vision-Based Relative Navigation for Formation Flying of Spacecraft", AIAA Guidance, Navigation, and Control Conference, Denver, CO, Aug. 14-17, 2000.

[4] H. Khalil, Nonlinear Systems, Prentice-Hall, Inc., 1996, pp. 115-116 and 626-629. 
[5] M. Krstic, I. Kanellakopoulos, and P. Kokotovic, Nonlinear and Adaptive Control Design, John Wiley \& Sons, Inc., 1995, p. 491.

[6] O. Egland and J.M. Godhaven, "Passivity-Based Adaptive Attitude Control of a Rigid Spacecraft", IEEE Transactions on Automatic Control, Vol. 39, No. 4, April 1994, pp. 842-846.

[7] B. Wie, Space Vehicle Dynamics and Contrl, AIAA Education Series, 1998, pp. 318-323.

[8] R.M. Sanner, "Adaptive Attitude Control Using Fixed and Dynamically Structured Neural Networks", AIAA Guidance, Navigation and Control Conference, San Diego, CA, July 29-31, 1996. 
